\title{
Protée
}

\section{Le miroir éclaté/A dança do existir. Altérité coloniale et violence de l'identité dans un solo de Vera Mantero}

\section{André Lepecki}

Volume 29, numéro 2, 2001

Danse et altérité

URI : https://id.erudit.org/iderudit/030627ar

DOI : https://doi.org/10.7202/030627ar

Aller au sommaire du numéro

Éditeur(s)

Département des arts et lettres - Université du Québec à Chicoutimi

ISSN

0300-3523 (imprimé)

1708-2307 (numérique)

Découvrir la revue

Citer cet article

Lepecki, A. (2001). Le miroir éclaté/A dança do existir. Altérité coloniale et violence de l'identité dans un solo de Vera Mantero. Protée, 29(2), 67-75. https://doi.org/10.7202/030627ar
Résumé de l'article

Dans cet essai, je me propose d'analyser le solo A Dança do Existir (1995) de Vera Mantero, chorégraphe portugaise, en montrant qu'il est une intervention culturelle dans la mémoire postcoloniale portugaise. La lecture des usages d'immobilité, du montage sonore et des déplacements chorégraphiques dans la pièce de Mantero permet d'examiner les notions d' " anesthésie culturelle » (Allen Feldman) et d'« actions immobiles » (Nadia Seremetakis) par rapport aux formations culturelles de la violence et de l'altérité dans la modernité européenne. 


\section{LE M IRO IR ÉCLATÉ/A DAN ÇA DO EXISTIR Altérité CO LO NIALE ET VIO Len CE DE L'IDENTITÉ DANS UN SO LO DE VERA MANTERO \\ Traduit de I'anglais par Jean-Pierre Vidal}

AN D RÉ LEPECKI

Treize années de guerre coloniale, l'effondrement soudain de l'empire, voilà deux événements qui semblaient bien destinés non seulement à produire un profond trauma dans nos consciences - semblable à celui d'une perte d'indépendance - mais aussi une profonde remise en question de la totalité de l'image que nous nous faisions de nous-mêmes et de notre reflet au miroir du monde. Mais au lieu de cela nous avons tous pu contempler cet étonnant spectacle: ni l'un ni l'autre ne se sont produits.

Eduardo Lourenço, O Labirinto da Saudade (nous soulignons)

Ne pas savoir

Ne pas lire

Ne pas savoir la moindre chose

Sur le monde.

Vera Mantero, premiers mots de A Dança do Existir

Ces deux épigraphes évoquent une double crise qui s'est produite dans le Portugal contemporain: une crise des sens et une crise de la mémoire. Elles rendent manifestes les termes dans lesquels la débâcle de cinq cents ans d'empire colonial a amené avec elle une profonde perturbation de l'image de l'identité nationale post-coloniale.

Je m'intéresse quant à moi à l'expressivité physique de ce corps postcolonial en crise qu'exhibe le Portugal. Je m'intéresse aux stratégies discursives de ce corps et plus particulièrement aux stratégies chorégraphiques par lesquelles la violence coloniale refoulée se trouve critiquée et portée sur la scène dans le Portugal contemporain. C'est l'un de ces moments, fait de critique intelligente et d'inquiétante mise au jour, qui forme le sujet de cet article: un solo de Vera Mantero intitulé A Dança do Existir (1995). Cette danse, comme nous le verrons, commence par montrer que la violence refoulée du passé colonial est toujours 
présente, avant de fouiller, d'illuminer et de subvertir cette même violence.

Eduardo Lourenço décrit la façon dont la fin brutale du colonialisme a été (et est encore) perçue collectivement au Portugal comme un non-événement, comme si les contrecoups de la guerre et les fissures tectoniques massives amenées par un changement social et politique considérable avaient conduit à l'effondrement plutôt qu'à l'émergence d'une énergie psychique et d'une force historique nationales. Pour Lourenço, cette fâcheuse situation de silence croupissant et d'apathie parait pour le moins intrigante. Comment est-il possible, se demande-t-il, qu'

un événement aussi spectaculaire que l'effondrement d'un "empire» vieux de cinq cents ans et dont la possession semblait si essentielle à la conservation de notre propre réalité historique et plus encore au maintien de notre propre image corporelle, éthique et métaphysique, en tant que Portugais, se soit déroulé sans le moindre drame [?] ${ }^{1}$

C'est de l'intérieur de cette «absence de drame» pour l'image corporelle des Portugais que l'on doit formuler une théorie des formes d'expression portugaises contemporaines, et tout particulièrement des formes d'art liées fondamentalement au corps, telle la danse.

La formule ironique "ne pas savoir, ne pas lire, ne pas savoir la moindre chose sur le monde" par laquelle Mantero ouvre A Dança do Existir reflète et répercute l'observation de Lourenço sur le nonévénement entouré d'un étonnant mutisme qu'aura été l'effondrement "soudain" de l'empire et la fin de la guerre coloniale. Ces mots soulignent aussi ce que l'anthropologue Allen Feldman nomme l'«anesthésie culturelle», qu'il définit comme

[...] the banishment of disconcerting, discordant and anarchic sensory presences and agents that undermine the normalizing and often silent premises of everyday life. ${ }^{2}$

Quels sont donc ces «présupposés tacites de la vie quotidienne» qui se trouvent écartés de la plupart des discours publics du Portugal contemporain? En général, ils concernent la (mauvaise) gestion mémorielle de la violence coloniale du pays et son absence de sensibilité à la résurgence récente de cette même violence à l'intérieur des frontières étroites de l'ex-métropole. En 1998, Eduardo Lourenço a souligné la façon dont les puissances de l'oubli et l'anesthésie culturelle exerçaient encore une répression collective massive du passé colonialiste, fasciste, dictatorial et violent du Portugal:

Ni en Italie, ni en Allemagne (avec la lourde croix que ce pays doit porter), ni même en Union Soviétique (la Russie

d'aujourd'hui) - tous pays où la tentation d'enfouir le passé sous une couche d'oubli a représenté une sorte de devoir ou de réflexe national - n'a-t-on pu observer l'émergence d'un tel phénomène de non-existence posthume. ${ }^{3}$

Non-existence, certes, mais seulement au niveau de ses manifestations conscientes ou publiques. Car les courants sous-jacents de violence et de colonialisme ont toujours été là; on peut les sentir, les goûter, les toucher, doucement, insidieusement, sous le voile de l'anesthésie culturelle et des discours publics de tolérance envers l'Autre: skin-heads de banlieue assassinant des Noirs, aussi bien à Lisbonne qu'à Porto; à l'intérieur du pays milices organisées de "bons" citoyens qui expulsent les gitans de leurs villages à la pointe du fusil, brutalités policières constantes à l'endroit des Noirs, tout ce compost pourrissant d'un racisme au quotidien. Sans oublier les éruptions occasionnelles de nostalgie coloniale qui, au début des années quatre-vingt-dix, ont notamment conduit à la mise en vente de cassettes vidéo contenant des scènes illustrant la vie nocturne «inoubliable» de l'Angola et du Mozambique des années cinquante et soixante. «Pour revivre le bon vieux temps» disait la publicité. En dépit d'un insistant discours public et gouvernemental sur la "tolérance raciale et culturelle» du Portugal, il suffit de s'attarder un peu au centre de Lisbonne, où des dizaines d'Africains se regroupent, chaque jour, sur les marches du Théâtre National, attendant d'être embauchés par des patrons blancs pour toutes sortes de petits boulots sous-payés, pour que bientôt les spasmes de la violence enfouie remontent le long de votre 
système nerveux, rendant enfin perceptible cette violence partout présente dans le silence collectif.

«Ne pas savoir, ne pas lire, ne pas savoir la moindre chose sur le monde». J'ai entendu la formule de Mantero pour la première fois en 1995: ces mots ouvraient sa danse de quinze minutes intitulée, de façon parfaitement appropriée, A Dança do Existir. Cette œuvre m'a fait une énorme impression. C'est une danse qui se déroule essentiellement dans l'obscurité, se manifeste surtout par le son, commence et se termine dans l'immobilité. Je ne l'ai vue qu'une seule fois sur scène, précisément le soir de la première et malgré son caractère très physique j'y suis revenu au fil des années uniquement par le son. Car, bien que la danse ait évidemment sa place dans ce solo, le souvenir que j'en garde est essentiellement acoustique. Tout au long des quinze minutes qu'il dure, le public peut voir la présence visuelle de Mantero subordonnée à une trame sonore complexe composée par Sérgio Pelágio et par Mantero elle-même. Pour moi, cette trame sonore est littéralement A Dança do Existir et je vois dans le déplacement qu'elle opère, de l'œil, organe habituellement consacré à l'appréciation de la danse, à l'oreille, une manifestation de résistance à l'anesthésie culturelle.

En suivant les déplacements, les substitutions et les manipulations sensoriels et mnémoniques opérés par la danse solo de Mantero, j'espère montrer ici comment elle critique et défie non seulement la perception que nous avons de la danse et sa théorie, mais, ce qui s'avère encore plus important, l'état collectif d'apathie culturelle et historique ainsi que l'anesthésie générale sensorielle et culturelle du Portugal contemporain.

A Dança do Existir exige un déplacement radical de l'optique tout en proposant d'entrée de jeu une écoute particulièrement intense - d'un corps de femme qui s'abandonne tout entière à un escamotage et à un dévoilement soigneusement chorégraphiés du passé collectif de violence coloniale, par l'immobilité, le mouvement et le son. L'œuvre commence par Mantero immobile, au bord de la scène à droite, près de la dernière rangée latérale de sièges. Elle présente une apparence plutôt bizarre. Un vieux t-shirt vert, tâché et à moitié déchiré couvre sa poitrine, une élégante robe de bal de soie bleue, à la mode des années cinquante, bouffe autour de sa taille, couvrant la majeure partie de ses jambes; un tutu blanc dépasse par endroits de sa robe et une vieille paire de chaussures de sport sales complète son accoutrement. Ses cheveux longs et emmêlés ne sont pas coiffés. Avec une concentration totale, elle écoute attentivement sa propre voix enregistrée qui décrit, sur le ton anodin de la conversation, ce qu'elle porte ce soir-là. Ce que nous découvrons alors, c'est que chaque pièce de son habillement, l'élégante comme la banale, la vieille comme la sale, transporte avec elle une histoire de violence délibérément cachée. La voix enregistrée de Mantero nous apprend en effet que la robe de bal vient de sa mère qui la portait dans les années cinquante dans les bals élégants de la haute société de Lisbonne. Ces bals étaient renommés pour leur chic et leurs excès contrastaient fortement avec les difficultés économiques de la majorité de la population: ils représentaient la façon qu'avait le régime de jouer la normalité sur un fond de misère noire et de répression. Le t-shirt, lui, vient des années d'adolescence de Mantero: elle aimait tant le porter que maintenant il tient à peine. S'il est déchiré, nous dit-elle, c'est parce qu'un ami «légèrement violent» l'a bousculée, une nuit, un peu plus fort que d'habitude et le lui a arraché. Les chaussures de sport sales appartenaient originellement à sa mère, nous apprend la voix off de Mantero. Un autre ami les avait données à sa mère mais elles étaient trop grandes pour elle. Sa mère les avait donc, à son tour, données à Mantero. Elles étaient également trop grandes pour elle, mais elle les gardait quand même parce qu'elle n'en avait pas d'autres du genre. Elle les avait emportées en Croatie où elle était allée voir un ami, pendant la guerre, en 1993. Il écrivait toute la journée et pendant ce temps-là, elle, elle travaillait. À la fin de la journée, ils allaient courir dans les champs. Certaines nuits, il lui semblait entendre les bombardements; elle rencontrait des réfugiés et des soldats encore sous le choc des bombardements. C'était un endroit 
formidable pour des vacances. Ses chaussures sont devenues rouges de la poussière et de la saleté rouillée typiques de cet endroit paisible, aux frontières de l'horreur, et elle ne les a jamais plus lavées. Ici, la voix enregistrée de Mantero arrête sa description et déclare: «en ce moment, je ne suis pas là». Et la scène devient complètement noire.

Dans cette obscurité, une nouvelle trame sonore commence à se faire entendre. Au lieu de la voix claire de Mantero, on entend une bande sonore complexe, exagérément verbale, hyperboliquement fragmentée, remplir les quatre coins du théâtre de son flot continu de voix. Il s'agit de la bande sonore qui commence par les mots: «ne pas savoir, ne pas lire, ne pas savoir la moindre chose sur le monde». Après l'immobilité initiale de Mantero écoutant attentivement sa propre narration des diverses violences qui se cachent dans des pièces de vêtement quotidien, cette bande sonore résonne fortement dans le noir et le public se retrouve dans la même position d'immobilité attentive que la danseuse vient de représenter sur la scène. Dans cette inversion spéculaire des rôles, c'est le moment pour le public de s'engager dans une archéologie de la violence quotidienne - qui n'est, somme toute, rien de plus qu'une archéologie des tensions entre l'identité et l'altérité. Tranquillement assis sur nos sièges, nous entrons dans nos propres danses d'exister. Et ce que nous écoutons n'a rien d'évident. Des voix différentes, des niveaux de langages différents (parfois poétique, parfois argotique, parfois religieux), différents accents s'entremêlent et sont ponctués de sons bizarres, de coupures brusques, d'interruptions musicales en tous genres. La verbosité de la bande sonore crée incontestablement un barrage sémantique et acoustique de forte intensité et le corps de Mantero semble effacé par cette masse linguistique et sonore écrasante qui, dans son montage complexe, flirte à la fois avec la signification et la dérision. Bien qu'il s'appuie fortement sur le langage, l'environnement sonore n'ouvre pas un monde de communication ou de représentation mais propose plutôt un langage de et dans la désarticulation, le court-circuit, l'association libre. La bande est faite de voix qui sont clairement «mises en scène» (celles de Mantero et de Pelágio qui jouent divers rôles en lisant des textes issus d'une grande variété de sources - poèmes, catalogues pornographiques, associations verbales se développant librement) alors que d'autres sont clairement «documentaires» (extraits d'émissions radiophoniques ou télévisuelles et d'entrevues faites à la radio ou dans la rue).

Tout au long des dix minutes que dure la bande sonore, nous entendons, entre autres choses: la voix de Pedro Paixão, un romancier portugais qui se pose des questions sur l'amour, Dieu, la mort et sa mère; sa voix se mêle à celles de Mantero et de Pelágio qui lisent la biographie de Glenn Gould, plus spécifiquement ce qui concerne ses phobies, son amour des structures, sa façon de chanter devant les animaux, au zoo; tout cela est entrelacé de voix de soldats traumatisés par les guerres coloniales, qui parlent de la misère du Portugal des années soixante et de la façon dont on les avait entraînés à tuer, rien qu'à tuer, en criant comme des fous que l'armée était magnifique, et comment on leur avait appris à se montrer fiers de leurs trophées macabres; et par-dessus cela, la voix d'un de ces anciens combattants qui se brise, sanglote et devient incapable de parler des tortures qu'il infligeait alors aux guérilleros africains; nous entendons alors en arrière-plan un extrait de $L a$ Passion selon saint Mathieu de Bach, le moment précis où le chœur implore la pitié de Dieu pour les larmes que nous avons fait couler; et tout cela est entremêlé çà et là d'un poème présentant un monologue intérieur délirant dit par une jeune femme dont la vie a commencé en Afrique puis s'est transportée au Brésil, après l'indépendance, avant de se retrouver au Portugal; et il y a encore d'autres voix, d'autres textes, d'autres textures qui viennent constamment s'ajouter à la bande, depuis des textes pornographiques jusqu'à l'éloge que fait William Blake de l'énergie considérée comme un éternel délice...

Après environ quatre minutes dans l'obscurité, la bande sonore arrive à un moment où l'on entend l'une après l'autre des douzaines de voix différentes prononçant simplement les mots «descobrir as regras». 
Des hommes, des femmes, aux accents différents, répétant tous cette phrase qui, en portugais, peut avoir des connotations légèrement différentes - "découvrir les règles» mais aussi «révéler les règles». De plus, le mot «regras» (règles) a également un double sens: il veut dire, comme en français, aussi bien «menstruations» que «norme». C'est à ce moment précis que la scène s'illumine brusquement d'une lumière vive qui nous révèle Mantero déjà en mouvement, pour la première fois, en plein centre de la scène. Avant de décrire ce qu'elle fait en bougeant, il est nécessaire de considérer tout ce qui a précédé ce moment d'illumination (littéralement) et de danse. Il est capital de considérer précisément maintenant le rôle du son et de l'immobilité qui ont servi en quelque sorte de préface à la découverte de Mantero par la lumière.

Ce qui frappe dans la première partie de A Dança do Existir, c'est l'accent vif et un peu argotique de la voix enregistrée de Mantero lorsqu'elle se heurte au contenu de ce qu'elle est en train de dire. Chaque pièce de vêtement qu'elle porte transporte avec elle non seulement une histoire, mais une histoire teintée d'actes de violence ou un contexte historique de violence. Sa silhouette se présente comme un composé de toutes ces histoires qui lui ont été transmises par des objets dont elle a hérité par sa mère, des objets détruits par des amants violents, des objets qui ont franchi les chemins poussiéreux de la transformation historique et de la guerre. Il n'est pas indifférent qu'elle écoute sa propre voix dans l'immobilité la plus complète et que cette immobilité s'étende au-delà des limites de son corps, dans la mesure où cette perception est transmise au public quand les lumières s'éteignent et qu'on devient brusquement parfaitement conscient qu'on est assis dans le noir à écouter d'étranges histoires d'amour et à entendre les voix de ceux qui se sont livrés à la violence coloniale. Quelle peut bien être l'intention de la stratégie chorégraphique de Mantero dans A

Dança do Existir, lorsqu'elle efface ainsi volontairement sa propre présence, qu'elle joue emphatiquement d'un déplacement sensoriel, du visible à l'invisible, qu'elle privilégie consciemment le son, le tout dans le contexte d'une ouvre qui est aussi, et explicitement, une critique postcoloniale des courants de violence qui courent sous la surface du Portugal contemporain?

Dans son essai sur l'anesthésie culturelle, Allen Feldman critique The Civilizing Process de Norbert Elias ${ }^{4}$. Pour Feldman, l'idée d'Elias selon laquelle «modernization entails the progressive withdrawal of violence from everyday life in tandem with its increasing monopolization by the state ${ }^{5}$ doit être remise en cause. Feldman donne l'exemple de plusieurs occurrences où l'État, par son intervention, «démocratise la violence», comme en Irlande du Nord et dans l'ex-Yougoslavie. Sous cet éclairage, la violence doit être replacée, «[from the] verges of civilizational processes and European modernity", au cœur même et de la civilisation et de la modernité ${ }^{6}$. C'est ce changement de perspective qu'illustre Mantero dans les histoires qu'elle raconte sur ce qui couvre son corps, dans sa danse d'exister. L'effet presque comique produit par sa première apparition dans son t-shirt déchiré, sa robe de bal en soie bouffante et ses chaussures de sport sales est littéralement miné par le récit qu'elle fait de la petite histoire de violence que renferme chaque partie de son vêtement. La violence sans merci du fascisme, la violence brutale des amants, la violence dévastatrice de la guerre, la violence normative de l'entraînement militaire ne sont pas présentées comme des événements extérieurs, échappant en quelque sorte au cours «normal» de l'histoire et de la quotidienneté, mais au contraire comme ce qui enferme la quotidienneté dans sa propre logique existentielle. On porte toujours la violence sur soi, même si l'on choisit de ne pas prêter l'oreille à ses éclats.

Et que dire de la stratégie chorégraphique que Mantero choisit de mettre en œuvre pour souligner, dans la dramaturgie même, la violence qui est au cœur de la quotidienneté? Pourquoi donc se tient-elle immobile? Pourquoi choisit-elle, précisément à ce moment d'auscultation historique, de ne pas bouger? Il serait utile d'évoquer ici la notion de "still act» [acte 
immobile] proposée par Nadia Seremetakis dans son importante critique de la place des sens dans la modernité 7 . Pour Seremetakis, le «still act» n'est pas une immobilisation du sujet dans la rigidité d'une statue mais plutôt un moment de disruption sociale au cours duquel le sujet suspend les hégémonies temporelles, narratives et idéologiques au moyen d'une tranquille interpellation de l'histoire. Pour Seremetakis, les «still acts» représentent ces moments de pause et d'arrêt dans lesquels le sujet - en produisant physiquement une rupture dans le flot de la temporalité - interpelle ce qu'elle appelle "historical dust» 8 .

En recourant à l'immobilité quand elle écoute sa propre voix dévoiler calmement la violence qui gît dans le banal, Mantero fait acte de résistance sensorielle. Plus important encore, cette résistance sensorielle en tant que re-organisation mnémonique se trouve ensuite transférée dans les corps du public. Car, après la disparition du corps de Mantero, c'est le public qui se voit plongé dans l'obscurité sous un véritable barrage sonore de voix. C'est le public qui assume alors le rôle actif de l'attention immobile.

En parcourant les notes prises par Mantero pour la création de A Dança do Existir, j'ai trouvé le passage suivant qui illustre bien ses idées sur l'utilisation du son, de l'obscurité et de l'immobilité dans sa danse d'exister:

Écouter un texte, des sons et de la musique dans l'obscurité c'est embarquer pour l'univers de l'invisible [...] Parler dans l'obscurité donne la possibilité de dire des choses qui impliquent les autres, c'est même avoir une chance de pénétrer dans leur monde intérieur, presque d'entrer dans leurs rêves comme un véritable personnage, plutôt que de leur donner quelque chose de plus visuel et de plus extérieur (à eux). ${ }^{9}$

En choisissant d'abord de ne pas danser et plutôt d'écouter, pour disparaître ensuite activement à la vue, Mantero résout une question chorégraphique concernant l'éthique de la remembrance et de la résistance des sens. Une question qui joue avec les possibilités offertes par le jeu de mots sur remembrance comme re-membrement, c'est-à-dire la re-organisation et la re-distribution du corps et de ses diverses parties, des sens et de leurs canaux, pour engendrer un nouveau corps imaginatif, créatif, provocateur et historiquement critique ${ }^{10}$.

Particulièrement révélatrice, dans cette stratégie, la suspicion dans laquelle la chorégraphe tient le visible et en particulier la capacité du visible de révéler la violence qui court sous l'écume des jours. Cette suspicion suggère de rectifier la notion de chorégraphie envisagée comme un art essentiellement visuel, ce qui entraîne une double conséquence théorique. Premièrement, cette pratique confirme l'opinion de Mark Franko selon laquelle lorsqu'on considère des danses qui se montrent "politiquement résistantes", on doit prendre en considération le fait que ce sont, très souvent, des pratiques asymétriques et non illustratives ${ }^{11}$. Pour Franko, cette dimension «non illustrative» des danses politiquement résistantes a des implications épistémologiques importantes, expressément la nécessité, pour les théoriciens et les historiens de la danse, de remettre en question «how much of dance practice materializes as visible, or should be understood in visual terms alone» ${ }^{12}$. Le questionnement de Franko s'applique tout particulièrement au cas qui nous occupe ici, dans la mesure où son projet d'élargir la fixation sur les sens dans les études de danse au-delà du champ étroit du visible (ce qu'il appelle la «visual fallacy in dance history", l'erreur de l'obsession visuelle dans l'histoire de la danse) illustre tout particulièrement la conception que se fait Mantero de l'utilisation du corps dans la danse. En 1993, deux ans avant la création de A Dança do Existir, Mantero avait déclaré, dans une interview au quotidien Público qu' «en ne se servant que du corps, la danse ne peut pas tout dire ${ }^{13}$. Pour Mantero, le corps est secondaire dans son œuvre, au sens où il doit savoir quand s'effacer pour dire les choses plus efficacement.

Dans ses nombreux niveaux, ses permutations et sa fragmentation stratégique, A Dança do Existir explore l'un des épisodes de l'histoire portugaise récente sur lesquels on a le plus fait silence, la guerre coloniale de 1961 à 1974 - une guerre qui a peut-être pris fin sur le 
plan de ses opérations militaires mais qui est encore présente dans la vie quotidienne du Portugal postcolonial. Ce qui s'avère le plus choquant pour le public portugais, c'est d'entendre les récits, presque jamais diffusés, des anciens combattants des guerres coloniales dont l'histoire, comme le relevait encore récemment Eduardo Lourenço en 1998, attend encore d'être racontée ${ }^{14}$.

Walter Benjamin, dans ses «Thèses sur la philosophie de l'histoire", nous dit qu'

[...] articulate the past historically does not mean to recognize it "the way it really was". It means to seize hold of a memory as it flashes up at a moment of danger. 15

Mantero articule une histoire qui «attend encore d'être contée» en créant une atmosphère d'urgence, dans l'obscurité et en donnant une voix à ceux qui ont été évacués de la mémoire historique du pays. Le souvenir revient par bribes, comme une articulation de l'histoire, grâce au va-et-vient urgent de la danseuse sur la scène, aux sons qui se heurtent en arrière-plan, et au caractère de témoignage que revêtent les déclarations des anciens combattants de la guerre coloniale telles que nous pouvons les entendre.

Il serait injuste de dire qu'il s'agit d'un solo sur la guerre coloniale. Cela reviendrait à réduire sa polysémie explosive et le caractère radicalement libérateur de ses ramifications fragmentées. Mais il est juste de dire que c'est un solo sur les forces qui sont à l'œuvre dans le Portugal contemporain et qui perpétuent les mêmes vieilles relations coloniales, les mêmes vieilles tensions raciales, recyclant et reénergisant ainsi, même si c'est de façon imperceptible, la violence sourde qui provient de ces années de guerre et de terreur. En ce sens, c'est un solo sur la nature répressive du silence tel qu'il infuse dans le corps social, lui donnant son système nerveux postcolonial ${ }^{16}$. C'est seulement sous cet éclairage que l'on peut comprendre pourquoi Mantero, dans un court texte publié dans le programme du soir de la première de A Dança do Existir, appelle son ouvre, en dépit de l'impact évident de sa masse sonore continue, «une danse de silence».
Nous pouvons maintenant comprendre ses raisons. Il s'agit d'une danse qui explore ce silence qui justement s'étend sous la verbosité, la visibilité et le bruit les plus assourdissants. Ce silence qui manifeste une "anesthésie culturelle», la capacité, selon Feldman, «to increase pain upon the Other [and] to render the Other's pain inadmissible to public discourse and culture" 17 .

Comment se fait-il que l'on écoute les voix censurées de l'histoire sous l'amnésie collective produite par l'éclatement du miroir colonial? Ici se pose, sous les auspices de l'aura benjaminienne, une question de chorégraphie - car cette écoute est aussi une façon de préparer le corps, de le mettre en condition, de l'accorder à une position spécifique dans le temps (historique) et l'espace (social et politique). Dans cette nouvelle position, le sujet négocie, valide et écarte ce qu'il considère appartenir au royaume de l'(in)signifiance et de l'(im)perceptibilité.

Pour Nadia Seremetakis, l'imperceptible a une structure sociale fondée sur des zones, culturellement prescrites, de non-expérience et de sens aboli.

Seremetakis identifie cette façon de faire taire et d'effacer les réalités sensorielles comme des indicateurs majeurs de moments de transformation sociale et historique. Ce silence imposé lance un défi à l'interprétation critique, en exigeant un nouveau réglage, une certaine mise au point des sens:

These moments [of historical transformation] can only be glimpsed at obliquely and at the margins, for their visibility requires an immersion into interrupted sensory memory and displaced emotions. ${ }^{18}$

La notion, avancée par Seremetakis, de regard oblique qui permet de s'immerger dans une «mémoire interrompue» ou une émotion déplacée (ou réprimée) s'avère capitale. Ces regards obliques représentent autant de regards déviants qui balaient les marges des sens et de l'histoire, ramassant, aux frontières du perceptible, des moments de silence collectif imposé et des traces de l'effacement continuel des expériences sensorielles et sociales. Il vaut la peine d'explorer ce chemin de traverse car il est celui qui convient le 
mieux, phénoménologiquement, à l'œuvre que nous considérons ici.

Après l'obscurité, une série de voix diverses prononce la formule "descobrir as regras", découvrir ou dévoiler les règles. C'est alors que la lumière envahit la scène et que nous découvrons Mantero en train de danser au beau milieu, dans son costume composite. C'est une danse fragmentée. Mieux, sa fragmentation semble exiger des regards perpétuellement obliques. La partie "danse» proprement dite de A Dança do Existir dure environ six minutes. C'est durant ces six minutes qu'émergent les voix les plus dérangeantes de la mémoire coloniale, avec l'ancien combattant qui sanglote, incapable de parler de son passé de tortionnaire. C'est là que nous entendons aussi les associations libres les plus hallucinées:

[Ancien combattant \#3] «Alors l'agression, je m'en souviens, elle est encore présente en moi, son agression sur ma mère...”. [Pedro Paixão] «Je veux dire, je suis déjà mort plusieurs fois».

[Ancien combattant \#3] «y compris un coup de pied qu'il m'a déjà donné une fois...».

[Mantero et Pelágio] «Fermez les yeux».

[Ancien combattant \#3] «... et cette vie misérable, la pauvreté, la misère, nous vivions tous dans la même pièce...”. [Mantero lisant un texte du poète Ruy Belo] «Dieu marche au bord de l'eau, avec le bas de ses pantalons relevés. Comment se couche un homme, comment il se lève. Nous sommes des enfants faits pour de grandes vacances". [Pelágio lisant un extrait de la biographie de Gould] «la notion qui veut que tous les sons soient dignes d'attention".

[Mantero murmurant très vite ]: «des amis qui ne se déçoivent pas les uns les autres, des rencontres ratées. Des rencontres. Des réfugiés. Au Nord. La faim. Le plaisir, la honte, le soleil. Au Brésil. Des événements importants. Pain, gymnastique, esprits. Désaveux, plaisir. Rencontres du troisième type. Tout faire pour ne pas tomber dans le trou. Fatigue, maux de tête, bonheur».

[Ancien combattant \#1] «... par exemple comme "rampez vers moi" et les gars qui rampent, "tout le monde rampe, rentrez-moi ces fesses, rentrez-moi ces culs, cette tête, rampez vers moi, vers moi, plus près!”. On était tous là, entassés les uns sur les autres, certains par-dessus, d'autres en dessous. "C'est bien, l'armée? Oui! L'armée, c'est magnifique! Allez, fils de putes, venez, rampez jusqu'à moi!"”.

Pendant ce temps-là, Mantero danse comme si son corps était discontinu. Elle garde le torse droit, se penchant rarement, ses bras et ses jambes tournent dans leurs articulations comme des pendules, à angles droits. Elle garde une grande concentration, comme si cette abstraction faisait partie d'une activité plus structurée, plus signifiante. Elle fait un pas de côté, elle ne reste jamais à la même place plus d'un instant. Il n'y a aucune fluidité dans son mouvement constant; tous ses mouvements sont faits de fragments discontinus, segmentés et isolés, exactement comme le mouvement d'une séquence filmique est fait d'images photographiques discontinues et fixes. Il est impossible d'en retirer une impression de complétude, d'avoir l'impression d'une danse complète, d'un corps complet. Mes regards sautent ici et là, essayant de suivre son corps et y renonçant la plupart du temps parce que mes oreilles veulent concentrer toute mon attention sur bande sonore éclatée et qu'on écoute mieux quand les yeux restent immobiles. Alors, je lance des regards obliques, encore et encore, vers la scène, manquant la plupart du temps le corps de la danseuse parce qu'entre-temps elle s'est déjà déplacée, elle est déjà ailleurs. Comme je l'ai déjà mentionné, le souvenir que je garde de cette représentation est exclusivement sonore. Et quand j'ai commencé à écrire ce texte, je n'arrivais pas du tout à me souvenir de la danse elle-même. J'ai donc demandé à Mantero de me fournir une bande vidéo de cette ouvre. Elle m'en a envoyé une, réalisée par un vidéaste professionnel. J'ai mis la bande dans mon magnétoscope et je l'ai laissée se dérouler. La nervosité de la caméra y est presque pathologique. Il n'y a pas une seule seconde où elle n'est pas en train de bouger autour du corps de Mantero, faisant des zooms in sur la scène, des zooms out vers la salle, capturant des parties de corps au hasard, tombant dans des séquences aléatoires, comme si elle cherchait, par ces 
zooms frénétiques, un corps qui, après tout, est là. Je suis sidéré quand je m'aperçois que même si Mantero ne court pas partout sur la scène, elle n'en échappe pas moins constamment à cet appareil optique qui tente de la capturer. Exactement comme elle l'avait promis au début de l'œuvre: «ce soir, je ne suis pas là . Ses mouvements discontinus anéantissent complètement l'effet documentaire mais abolissent aussi l'effet de distraction que la danse a eu sur mon inconscient optique. L'enregistrement vidéo de A Dança do Existir de Mantero est une réplique mimétique du manifeste sensoriel radical qu'est cette danse.

Le solo de Mantero constitue un moment de suspens historique, un instant d'interruption, de disruption, un moment qui vient illuminer les marges d'un champ répressif de silence et de poussière historiques. Le catalyseur de ma quête oblique au milieu de ces images, de ces perceptions et de ces émotions déplacées aura été une danse dont je me suis souvenu essentiellement comme d'un ensemble de sons. La danse: chambre d'écho où la mémoire, le mouvement et la violence se heurtent, donnant forme aux corps, chorégraphiant les identités, redistribuant les altérités.

\section{N O TES}

1. E. Lourenço, O Labirinto da Saudade. Psicanálise mítica do destino português, Lisbonne, Publicações Dom Quixote, 1991, p. 43. Toutes les citations portugaises ont été traduites à partir de l'anglais.

2. A. Feldman, «From Desert Storm to Rodney King via exYugoslavia: On cultural Anesthesia ", dans The Senses Still: Perception and Memory as material Culture in Modernity, N. Seremetakis (dir.), Chicago, The University of Chicago Press, 1996, p. 89 [l'exclusion des présences et des agents sensoriels déconcertants, discordants et anarchiques qui minent les présupposés normalisateurs et souvent tacites de la vie quotidienne.]

3. E. Lourenço, Portugal como Destino seguido de Mitologia da Saudade, Lisboa, Gradiva, 1999, p. 67-68 (c'est moi qui traduis).

4. N. Elias, The Civilizing Process, New York, Urizen Books, 1978.
5. Feldman, op. cit., p. 87. [la modernisation entraîne la disparition progressive de la violence dans la vie quotidienne coïncidant avec sa monopolisation de plus en plus grande par l'État].

6. Ibid. [des confins du processus de civilisation et d'engendrement de la modernité européenne.]

7. N. Seremetakis, "The Memory of the Senses, Part II: Still Acts", dans The Senses Still. Perception and Memory as Material Culture in Modernity, N. Seremetakis (dir.), Chicago, The University of Chicago Press, 1996, p. 23-43.

8. Ibid, p. 12. La notion de "poussière de l'histoire» vient de Walter Benjamin. Pour Benjamin, la nature de la société du confort a été parfaitement captée dans l'image des ruines, d'où l'importance de la poussière. La poussière, en tant qu'image dialectique, montre comment la sédimentation imperceptible des événements historiques anesthésie les sens, dans un tranquille processus collectif de répression, lui-même en forme de sédimentation. Pour Benjamin, «L'histoire est si immobile qu'elle s'empoussière " écrit Susan Buck-Morss - mais aussi, pourrait-on ajouter, l'histoire produit de la poussière pour pouvoir offrir le spectacle affairé du progrès. Le Portugal contemporain étouffe sous ces deux avatars de la poussière historique.

9. V. Mantero, notes chorégraphiques pour la création de A Dança do Existir, carnet intitulé «O meu trabalho" [mon travail] (c'est moi qui traduis).

10. Sur les implications théoriques de cette re/membrance, en particulier dans la danse contemporaine, cf. G. Brandstetter et $\mathrm{H}$. Völckers (dir.), Remembering the Body, Ostfildern-Ruit, Hatje Cantz Publishers, 2000.

11. M. Franko, Dancing Modernism/Performing Politics, Bloomington \& Indianapolis, Indiana University Press, 1995, p. XII.

12. Ibid. [l'importance de ce qui, dans la pratique de la danse, se matérialise comme visible, ou qui devrait être compris uniquement en termes visuels].

13. M. J. Fazenda, «Apenas com o corpo, a dança não consegue dizer tudo ", Público, 15 octobre 1993, p. 28 (c'est moi qui traduis).

14. E. Lourenço écrit: «Tout au long des treize années de guerres coloniales en Guinée, en Angola, au Mozambique, des milliers d'étudiants, des médecins, des intellectuels ont été mobilisés pour la dernière et absurde croisade menée contre les mouvements africains d'indépendance. L'histoire de cette mobilisation massive [...] n'a pas encore été écrite». Voir Lourenço, 1999, p. 69 (c'est moi qui traduis). 15.W. Benjamin, "Theses on the Philosophy of History", dans Illuminations, H. Arendt (dir.), New York, Shocken Books, 1969, p. 222 [... articuler historiquement le passé ne veut pas dire le reconnaître " tel qu'il a vraiment été». Cela veut dire se saisir d'un souvenir quand il revient en un éclair, au moment d'un danger.]

16. Cf. M. Taussig, The Nervous System, New York et Londres, Routledge, 1992, en particulier l'introduction et les chap. 7 et 8 .

17. Cf. Feldman, op. cit., p. 90 [... d'accroître la douleur de l'Autre (et) de rendre cette douleur inadmissible pour le discours public et la culture officielle.]

18. Seremetakis, op. cit., p. 23. [Ces moments (de transformation historique), on ne peut que les entrevoir obliquement et dans les marges, car les voir exige une immersion dans une mémoire des sens interrompue et dans des émotions déplacées]. 